\title{
Association of Fecal Microflora with Bronchiolitis
}

\author{
Seema Rai ${ }^{1} \cdot$ Amarpreet Kaur $^{1} \cdot$ Gurmeet Kaur Sethi $^{1} \cdot$ Jenny Goyal ${ }^{1} \cdot$ Savneet Kaur ${ }^{1} \cdot$ Hobinder Arora $^{1}$
}

Received: 31 January 2019 / Accepted: 8 March 2019 / Published online: 30 March 2019

(C) Dr. K C Chaudhuri Foundation 2019

To the Editor: The gut microbial community is known to affect respiratory diseases like asthma and cystic fibrosis [1, 2]. We conducted this study to evaluate the association of gut microflora with bronchiolitis and to determine whether it affects the severity of bronchiolitis. Thirty cases of bronchiolitis along with 60 healthy controls were enrolled in the study. We collected the stool specimens, did stool culture from the cases and controls, and identified the organisms. Among the total cases (30), E. coli was found in $20(66.7 \%)$, none had respiratory difficulty and it was statistically significant $(p<0.05)$; Klebsiella in $9(30.0 \%)$ and all had respiratory difficulty which was statistically significant $(p<0.05)$; and Citrobacter in $1(3.3 \%)$, who had respiratory difficulty, as shown in Table 1. Among the controls, these proportions were $66.7 \%$ for E. coli (40 of 60$), 1.7 \%$ for Klebsiella ( 1 of 60 ) and $31.7 \%$ for Citrobacter (19 of 60) subjects. In the present study, isolation of Klebsiella significantly affected the development of bronchiolitis as compared to the findings in the study by Hasegawa et al., in which bacteroides dominant isolates had more severe bronchiolitis [3]. In the Escherichia coli dominant group, the likelihood of having bronchiolitis was not significantly different in the case and control group. Sjögren et al. found that bacteroid dominant infants had lower level of Toll-like receptor and inflammatory cytokines in the peripheral blood [4]. They proposed common mucosal response (antigen presentation at one mucosal site also affects the responsiveness of other mucosal sites). Ichinohe et al. reported that a disruption of gut microbiota (i.e., dysbiosis) impairs the antibody responses after influenza virus infection, suggesting the need for intact commensal bacteria [5]. The present study concluded that there is an association between bronchiolitis and gut microflora and presence of Klebsiella in the gut associates with respiratory difficulty among cases of bronchiolitis.

Seema Rai

seemadoc98@yahoo.co.uk

1 Department of Pediatrics, Guru Gobind Singh Medical College, Sadiq Road, Faridkot, Punjab 151203, India
Table 1 Distribution of cases with respect to respiratory difficulty $(n=30)$

\begin{tabular}{clll}
\hline & \multicolumn{2}{c}{ Respiratory difficulty } & \multirow{2}{*}{ Statistical significance } \\
\cline { 2 - 3 } Organism & Present $(n=10)$ & Absent $(n=20)$ & \\
\hline E. coli & & & \\
Present & 0 & 20 & $<0.05^{\#}$ \\
Absent & 10 & 0 & \\
Klebsiella & & 0 & $<0.05^{\#}$ \\
Present & 9 & 20 & \\
Absent & 1 & 0 & $>0.05^{\#}$ \\
Citrobacter & & 0 & \\
Present & 1 & 20 & \\
Absent & 9 & & \\
\hline
\end{tabular}

${ }^{\#}$ Fisher Exact test

\section{Compliance with Ethical Standards}

Conflict of Interest None.

\section{References}

1. Bruzzese E, Callegari ML, Raia V, et al. Disrupted intestinal microbiota and intestinal inflammation in children with cystic fibrosis and its restoration with Lactobacillus GG: a randomised clinical trial. PLoS One. 2014;9:e87796.

2. Nakayama J, Kobayashi T, Tanaka S, et al. Aberrant structures of fecal bacterial community in allergic infants profiled by $16 \mathrm{~S}$ rRNA gene pyrosequencing. FEMS Immunol Med Microbiol. 2011;63: 397-406.

3. Hasegawa K, Linnemann RW, Mansbach JM, Ajami JN, Espinola JA, Petrosino J. The fecal microbiota profile and bronchiolitis in infants. J Pediatr. 2016;138:1-9.

4. Sjögren YM, Tomicic S, Lundberg A, et al. Influence of early gut microbiota on the maturation of childhood mucosal and systemic immune responses. Clin Exp Allergy. 2009;39:1842-51.

5. Ichinohe T, Pang IK, Kumamoto Y, et al. Microbiota regulates immune defense against respiratory tract influenza A virus infection. Proc Natl Acad Sci U S A. 2011;108:5354-9.

Publisher's Note Springer Nature remains neutral with regard to jurisdictional claims in published maps and institutional affiliations. 